\title{
Acute Displaced Fracture of Lateral Acromion after Reverse Shoulder Arthroplasty: A Case Report and Surgical Technique
}

\author{
Chul-Hyun Cho, Jae-Won Jung, Young-Jae Lim, Sang-Soo Na, Du-Han Kim ${ }^{\square}$ \\ Department of Orthopedic Surgery, Dongsan Medical Center, School of Medicine, Keimyung University, Daegu, Korea
}

\begin{abstract}
Acromial fractures are well-documented complications subsequent to reverse shoulder arthroplasty (RSA), and most appear as stress fractures with no history of single trauma. To date, no study has reported the occurrence of acute displaced acromial fracture due to sudden strong deltoid contraction during heavy work. Displacement of the fracture results in a challenging surgery since it is difficult to obtain adequate fixation in thin and osteoporotic bones. We report a rare case of acute displaced acromial fracture after successful RSA treatment, using a novel technique of open reduction and internal fixation, applying two $4.5 \mathrm{~mm}$ cannulated screws and lateral clavicle precontoured plate.
\end{abstract}

(Clin Shoulder Elbow 2019;22(2):106-109)

Key Words: Fractures; Scapula; Acromion; Reverse shoulder arthroplasty

Reverse shoulder arthroplasty (RSA) is a revolutionary treatment option for debilitating shoulder diseases. Although studies demonstrate a satisfactory clinical outcome following RSA, there are numerous reports on accompanying complications. ${ }^{1-3)}$

Acromial fractures are well-documented complications subsequent to RSA, with reported incidences ranging from $0.9 \%$ to $10.2 \% .^{3-6)}$ Because of the increased arm length and deltoid tension after RSA, there is an increase in force at the deltoid muscle origin sites. Hence, most postoperative RSA acromial fractures appear as stress fractures rather than acute fractures. Patients who experience this complication have inferior clinical outcomes and may be at increased risk for revision surgery as compared to patients without acromial fracture. ${ }^{4-6)}$ However, there is currently no consensus on the appropriate treatment of acromial fractures after RSA. ${ }^{4)}$ Furthermore, there are numerous controversies regarding the outcomes in patients with acromial fractures who were treated with conservative or operative management. $^{1,4,5,7)}$ The operative treatment of displaced acromial fracture after RSA is challenging because it is difficult to obtain adequate fixation in thin and osteoporotic bones. ${ }^{7)}$

In the current study, we report a rare, successful postoperative surgically treated case of acute displaced acromial fracture after RSA. We also describe a novel technique of open reduction and internal fixation using two $4.5 \mathrm{~mm}$ cannulated screws and a lateral clavicle pre-contoured plate.

\section{Case Report}

A 63-year-old, right-hand dominant carpenter, presented at our clinic in November 2014 with chief complaint of right shoulder pain over a 2-month duration. The patient was taking anticoagulant medication due to pulmonary thromboembolism. Magnetic resonance imaging (MRI) diagnosed complete tear of the supraspinatus tendon and the subscapularis tendon, following which a rotator cuff repair was performed. After 2 years, the patients visited the outpatient clinic for sudden right shoulder pain while carrying heavy load. Physical examination revealed the active range of motion (ROM) was $90^{\circ}$ of forward flexion,

Received January 17, 2019. Revised March 5, 2019. Accepted March 19, 2019.

Correspondence to: Du-Han Kim

Department of Orthopedic Surgery, Dongsan Medical Center, School of Medicine, Keimyung University, 56 Dalseong-ro, Jung-gu, Daegu 41931, Korea

Tel: +82-53-250-8028, Fax: +82-53-250-7205, E-mail: osmdkdh@gmail.com, ORCID: https://orcid.org/0000-0002-6636-9340

IRB approval (exemption granted): Keimyung University Dongsan Medical Center (No. 2018-11-017).

Financial support: None. Conflict of interests: None. 
$80^{\circ}$ of abduction, $40^{\circ}$ of external rotation, and internal rotation of the buttock level. MRI findings disclosed complete retear of the supraspinatus and subscapularis tendons that were retracted to the level of the glenoid. After deliberating the risks and benefits of partial repair, superior capsular reconstruction, tendon transfer, and RSA in the current scenario, we concluded that he was a good candidate for RSA, which was subsequently performed by applying the Trabecular Metal Reverse Shoulder System (Zimmer, Warsaw, IN, USA). During the procedure, we observed that there were no preoperative acromial pathologies (thinning, erosion, os acromiale, etc.).

The patient reported satisfactory pain relief and functional restoration after RSA. At the postoperative 4 weeks follow-up, his active ROM increased to forward flexion of $140^{\circ}$, abduction of $120^{\circ}$, external rotation of $45^{\circ}$, and internal rotation of the third lumbar vertebra. The patient returned to work of his own will. At 4 months after RSA, the patient returned to the outpatient clinic with sudden onset of right shoulder pain accompanied with a popping sound, whilst work with a drill. The patient denied any pain and disability of the right shoulder before this event. Plain radiographs and computed tomography scan revealed a displaced acromial fracture extending from the posterior to the acromioclavicular joint (Fig. 1). Considering the fracture location, displacement, age, activity, and occupation, an open reduction and internal fixation was planned.

After administering general anesthesia, the patient was placed in the beach chair position. Vertical skin incisions, known as sabre incisions, were made along the lateral margin of the acromion and scapular spine. The fracture site was exposed after raising the fasciocutaneous flap medially along the incision line.
A periosteal elevator was used to lift the angulated anterior fragment, and the fracture fragments were reduced using a reduction clamp. Provisional fixation was implemented with a K-wire and clamp. Two $4.5 \mathrm{~mm}$ full threaded cannulated screws were fixed to the perpendicular direction of the fracture line. This was followed by plate fixation, where a lateral clavicle pre-contoured plate (Acumed, Hillsboro, OR, USA) was bent along the acromial bony contour (Fig. 2). Postoperatively, the shoulder was immobilized with an abduction brace for 6 weeks. Passive ROM exercises commenced from 4 weeks after surgery, and active ROM exercise and normal daily use of the affected shoulder was encouraged at 8 weeks after surgery. No postoperative complications occurred.

At 18 months after fracture fixation, the fracture was completely healed. The patient was satisfied with his shoulder status and returned to work (Fig. 3). He was capable of $140^{\circ}$ of forward flexion, $120^{\circ}$ of abduction, $50^{\circ}$ of external rotation, and the third lumbar vertebra level of internal rotation. His visual analogue scale for pain, American Shoulder and Elbow Surgeons score, and Subjective Shoulder Value were 0\%, 85\%, and 80\%, respectively.

\section{Discussion}

The main principle of RSA is to transfer the center of rotation of the glenohumeral joint distally and medially. By increasing the deltoid lever arm, stress can be loaded to the acromion, the primary originating site of the deltoid muscle. ${ }^{1)}$ Most acromial fractures after RSA appear as stress fractures with no history of any trauma, and occur at variable locations along the acromion
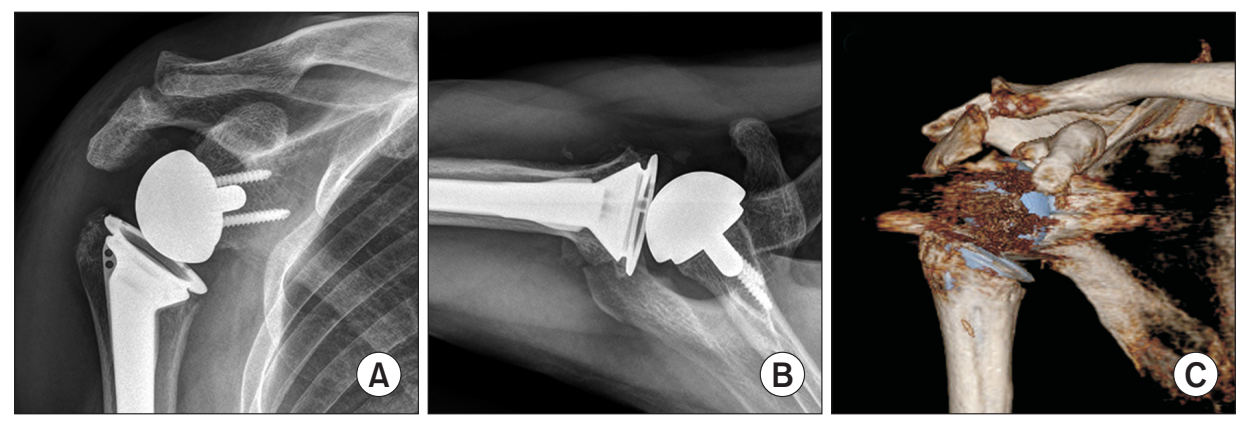

Fig. 1. Plain radiographs (A, B) and 3-dimensional computed tomography (C) scan show the displaced acromial fracture through the posterior to the acromioclavicular joint.
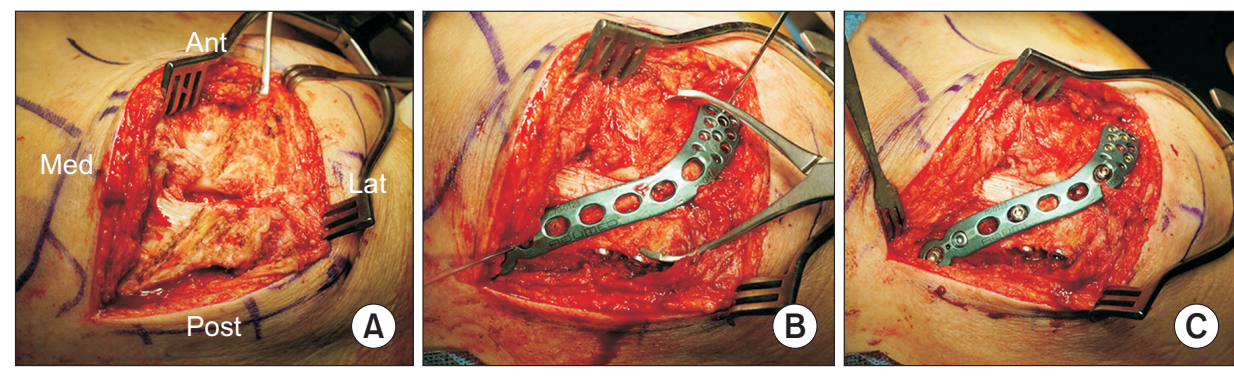

Fig. 2. Intraoperative photos showing sequential steps of open reduction and internal fixation of the displaced acromial fracture. (A) Reduction of the fracture using periosteal elevator and reduction clamp. (B) Fixation of two $4.5 \mathrm{~mm}$ cannulated screws. (C) Fixation of a lateral clavicle pre-contoured plate.

Ant: anterior, Med: medial, Lat: lateral, Post: posterior. 

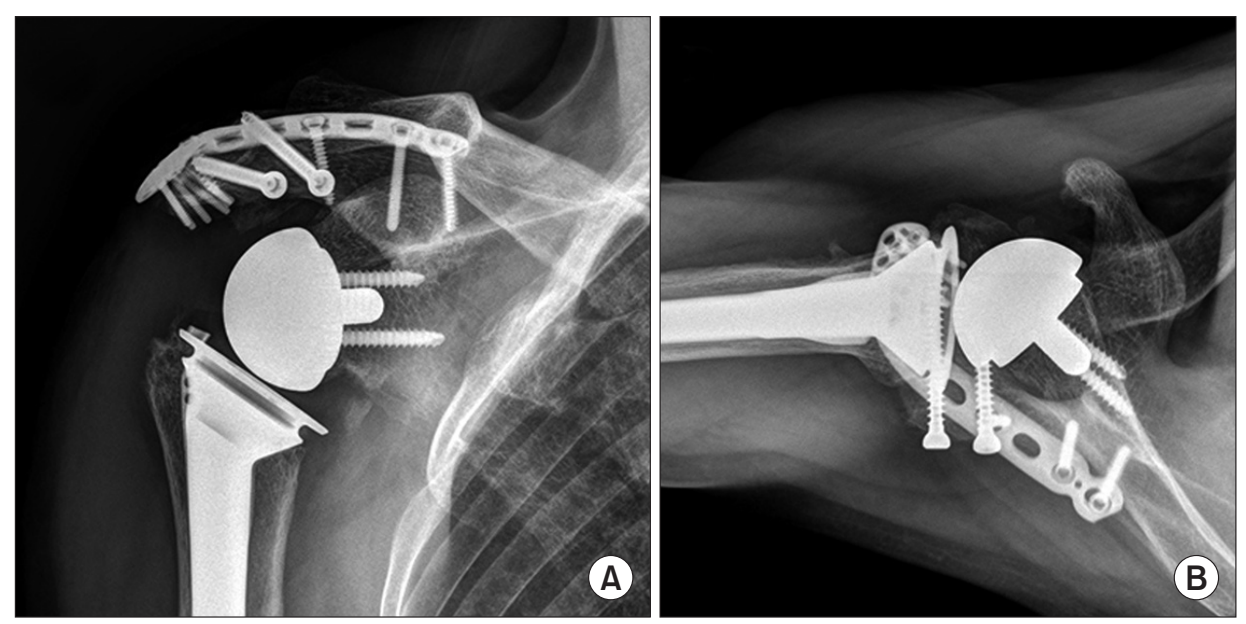

Fig. 3. (A, B) Plain radiographs at 18 months after fixation showing complete healing of the fracture. and scapular spine. ${ }^{3)}$ In our case, the patient was a relatively young, active, and busy worker. The patient decided to return to work 4 weeks after RSA and denied any pain and disability of the right shoulder before the sudden onset of pain with popping sound while using a drill at work 4 months after RSA. The sudden, strong deltoid contraction induced while using a drill was thought to be the main cause of the acute acromial fracture. To the best of our knowledge, there has been no report for acute acromial fracture occurring by this mechanism.

Acromial fractures after RSA have variable presentations, ranging from non-displaced to displaced fractures. Although several studies report that postoperative acromial fractures have negative effects on final outcomes, the management of postoperative acromial fractures is not well established. ${ }^{4-7)}$ Gerber et al. ${ }^{2)}$ advocated for surgical treatment of scapular spine or acromial base fractures, and conservative treatment for the more lateral acromial fractures. Non-displaced fractures detected early can be treated conservatively, but the management of displaced fractures is challenging for the surgeon because of high rates of malunion or nonunion; also, decreased functional acromial fractures after RSA are challenging to treat because of the complex 3-dimensional anatomy, poor bone stock, and variation in fracture patterns, each of which makes stable fixation difficult. ${ }^{8)}$

When open reduction and internal fixation is the preferred mode for treating displaced lateral acromion fractures, the goal is to apply compression at the fracture site and neutralize the distraction forces of the deltoid." To obtain adequate fixation, several techniques (tension bands, mesh plates, cruciform pilon plates, and locking plates) have been introduced. ${ }^{6-10)}$ The previously described tension band-fixation technique resulted in poor functional results for postoperative acromial fractures. ${ }^{6,7)}$ This fixation method may be insufficient to withstand the forces generated by the deltoid muscle. Rouleau and Gaudelli ${ }^{10)}$ described a dual locking plate fixation technique that provides rigid fixation for acromial base or scapular spine fractures. However, this technique cannot be applied to treat lateral or far distal acromial fractures.

In our current case, the fracture was displaced through the posterior to the acromioclavicular joint, and was classified as an unstable type-Il fracture by the classification of Crosby et al. ${ }^{3)}$ Because of the distal location of the fracture, it was more difficult to get sufficient bony purchase with standard plate and screws. Before plate fixation, two $4.5 \mathrm{~mm}$ cannulated screws were fixed along the perpendicular direction of the fracture line to resist the tensile force of the deltoid. These screws help to sustain the anatomical reduction, and prevent fixation failure by angulation of distal fragment. It also allows for compression along the fracture site and resists motion in all directions. This was followed by bending and fixation of the lateral clavicle pre-contoured plate along the bony contour of the acromion. To improve fixability, a lateral clavicle pre-contoured plate is advantageous in that it is possible to apply more locking screws on the tip of acromion as compared to other plates. We believe that our fixation technique provides sufficient stability for displaced lateral acromion fractures following RSA.

\section{References}

1. Boileau P, Watkinson DJ, Hatzidakis AM, Balg F. Grammont reverse prosthesis: design, rationale, and biomechanics. J Shoulder Elbow Surg. 2005;14(1 Suppl S):147S-61S. doi: 10.1016/ j.jse.2004.10.006.

2. Gerber C, Pennington SD, Nyffeler RW. Reverse total shoulder arthroplasty. J Am Acad Orthop Surg. 2009;17(5):284-95.

3. Crosby LA, Hamilton A, Twiss T. Scapula fractures after reverse total shoulder arthroplasty: classification and treatment. Clin Orthop Relat Res. 2011;469(9):2544-9. doi: 10.1007/s11999011-1881-3.

4. Levy JC, Anderson C, Samson A. Classification of postoperative acromial fractures following reverse shoulder arthroplasty. J Bone Joint Surg Am. 2013;95(15):e104. doi: 10.2106/JBJS. K.01516. 
5. Walch G, Mottier F, Wall B, Boileau P, Molé D, Favard L. Acromial insufficiency in reverse shoulder arthroplasties. J Shoulder Elbow Surg. 2009;18(3):495-502. doi: 10.1016/j.jse. 2008.12.002.

6. Wahlquist TC, Hunt AF, Braman JP. Acromial base fractures after reverse total shoulder arthroplasty: report of five cases. J Shoulder Elbow Surg. 2011;20(7):1178-83. doi: 10.1016/ j.jse.2011.01.029.

7. Mayne IP, Bell SN, Wright W, Coghlan JA. Acromial and scapular spine fractures after reverse total shoulder arthroplasty. Shoulder Elbow. 2016;8(2):90-100. doi: 10.1177/ 1758573216628783.
8. Camarda L, Phadnis J, Clitherow H, Bain G. Mesh plates for scapula fixation. Tech Shoulder Elb Surg. 2015;16(3):79-84. doi: 10.1097/BTE.0000000000000054.

9. Hess F, Zettl R, Smolen D, Knoth C. Anatomical reconstruction to treat acromion fractures following reverse shoulder arthroplasty. Int Orthop. 2018;42(4):875-81. doi: 10.1007/s00264017-3710-0.

10. Rouleau DM, Gaudelli C. Successful treatment of fractures of the base of the acromion after reverse shoulder arthroplasty: Case report and review of the literature. Int J Shoulder Surg. 2013;7(4):149-52. doi: 10.4103/0973-6042.123531. 